Res. 21:3405-3411.

4.Diatchenko, L., Y.F. Lau, A.P. Campbell, A. Chenchik, F. Moqadam, B. Huang, S. Lukyanov, K. Lukyanov et al. 1996. Suppression subtractive hybridization: a method for generating differentially regulated or tissue-specific cDNA probes and libraries. Proc. Natl. Acad. Sci. USA 93:6025-6030.

5.Gauchat, J.F., J.P. Aubry, G. Mazzei, P. Life, T. Jomotte, G. Elson and J.Y. Bonnefoy. 1992. Human CD40-ligand: molecular cloning, cellular distribution and regulation of expression by factors controlling IgE production. FEBS Lett. 315:259-266.

6.Hamilton, B.A., W.N. Frankel, A.W. Kerrebrock, T.L. Hawkins, W. Fitzhugh, K. Kusumi, L.B. Russell, K.L. Mueller et al. 1996. Disruption of the nuclear hormone receptor $\mathrm{ROR} \alpha$ in staggerer mice. Nature 379:736739.

7.Liang, P. and A.B. Pardee. 1992. Differential display of eukaryotic messenger RNA by means of the polymerase chain reaction. Science 257:967-970.

8.Lim, C., N. Jabrane-Ferrat, J.D. Fontes, H. Okamoto, M.R. Garovoy, B.M. Peterlin and C.A. Hunt. 1997. Sequence-independent inhibition of RNA transcription by DNA dumbbells and other decoys. Nucleic Acids Res. 25:575-581.

9.Morishita, R., G.H. Gibbons, M. Horiuchi, K.E. Ellison, M. Nakajima, L. Zhang, Y. Kaneda, T. Ogihara and V.J. Dzau. 1995. A gene therapy strategy using a transcription factor decoy of E2F binding site inhibits smooth muscle proliferation in vivo. Proc. Natl. Acad. Sci. USA 92:5855-5859.

10.Yamada, T., M. Horiuchi, R. Morishita, L. Zhang, R.E. Pratt and V.J. Dzau. 1995. In vivo identification of a negative regulatory element in the mouse renin gene using direct gene transfer. J. Clin. Invest. 96:1230-1237.

The authors thank Drs. Ed Zanders and Mike Trower (Glaxo Wellcome, Stevenage, England, UK) for providing the high-density array cDNA library and assistance in the software analysis of hybridization data. Address correspondence to Markus Steinmayr, Geneva Biomedical Research Institute, Glaxo Wellcome Research and Development SA, 14 chemin des Aulx, 1228 Planles-Ouates, Switzerland.

Received 27 February 1997; accepted 24 June 1997.

Markus Steinmayr, JeanPierre Aubry and Michael Becker-André

Geneva Biomedical Research Institute

Glaxo Wellcome Research and

Development $S A$

Plan-les-Ouates, Switzerland

\section{Cosmid Vector for the Cloning and Study of Complex GC-Rich Genomes}

BioTechniques 23:812-814 (November 1997)

Cosmid vectors are designed to accommodate large DNA fragments (35-45 kbp) and are thus routinely used to generate genomic libraries that enable the assessment of the linkage between genes by chromosome walking (3). However, due to the large size of inserts within cosmid recombinants, these cannot be easily released in their intact form from the vector because of the absence of suitable restriction sites in the multiple cloning site (MCS). We are presently involved in the generation of a bovine herpesvirus 1 (BHV-1) cosmid-based system representing the whole viral genome within overlapping fragments that would be capable of re- generating infectious virus following homologous recombination by cotransfection of host cells. To prevent recombination between vector sequences that would result in the insertion of these within the viral genome, the regeneration of infectious virus from such a system requires the a priori excision of full-length DNA inserts from the vector. Since the GC content of the BHV-1 genome is exceptionally high (72\%) (2), we modified the commercial cosmid vector SuperCos 1 (Stratagene, La Jolla, CA, USA) so as to flank the BamHI cloning site with two octanucleotide restriction sites consisting of only A and T residues, SwaI and PacI, creating SuperCos/HPS (HPS = HindIII, PacI and $S w a I)$. Preliminary analyses have shown that BHV-1 DNA is not cleaved by $S w a$ I and is restricted only once with PacI.

To construct the SuperCos/HPS vector, we designed two phosphorylated and complementary oligonucleotides containing HindIII, PacI, SwaI, DraI

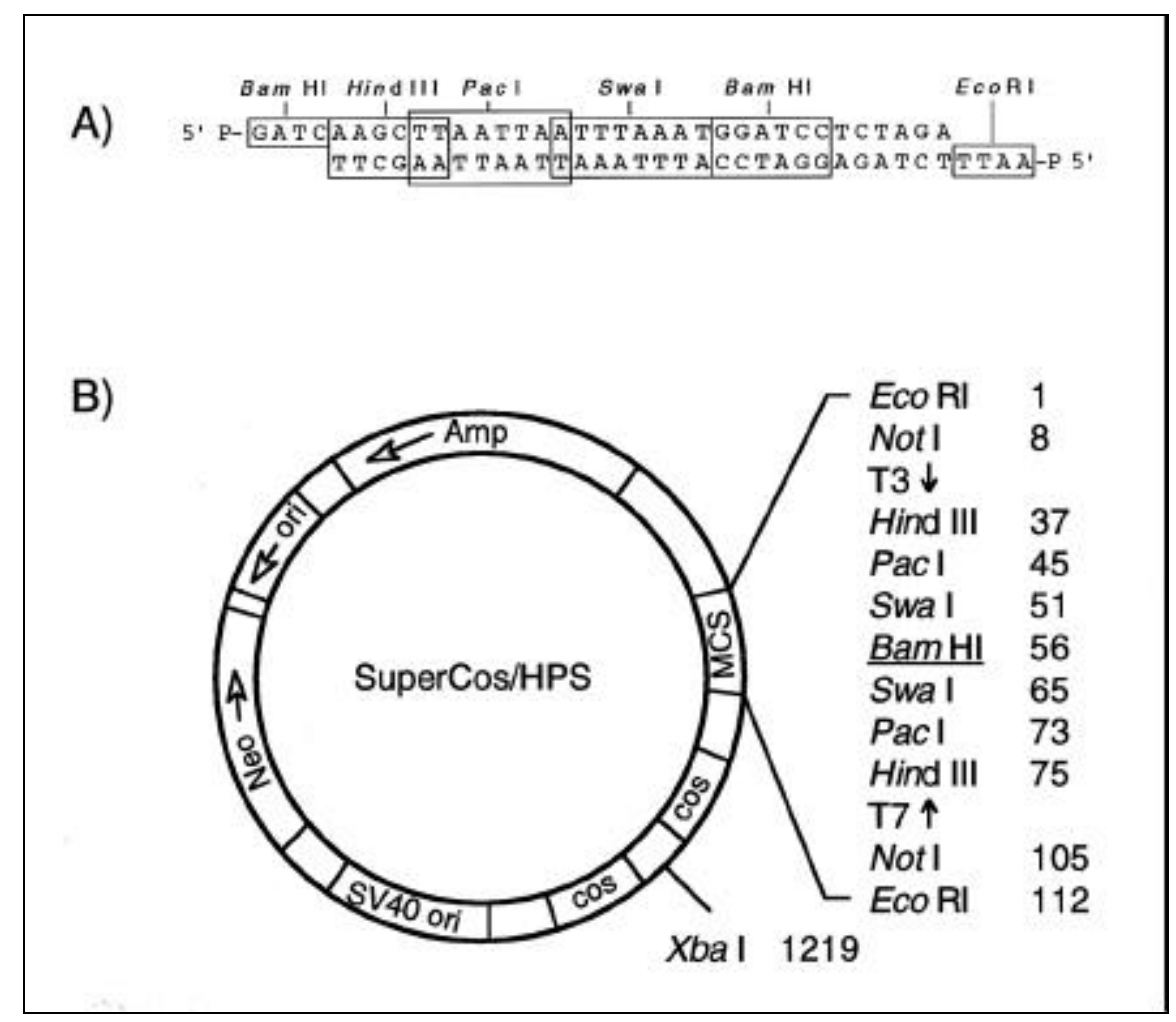

Figure 1. Characteristics of the SuperCos/HPS vector. (A) The adapter that was used for the modification of the SuperCos 1 vector consisted of two phosphorylated and complementary 35-base oligonucleotides containing HindIII, PacI, SwaI and BamHI restriction sites (boxed in) as well as non-regenerating compatible BamHI and EcoRI cohesive ends. (B) Schematic representation of the 7986-bp SuperCos/HPS vector illustrating the restriction sites of the MCS and their corresponding positions; the BamHI cloning site is underlined. Note that a third HindIII site is located at position 3422 of the vector. 


\section{Benchmarks}

(occurring within SwaI) and BamHI restriction sites as well as compatible non-regenerating BamHI and EcoRI cohesive ends (Figure 1A). The oligonucleotides were ligated in the presence of T4 DNA ligase to the SuperCos 1 vector previously digested with BamHI and dephosphorylated. The DNA that had been subsequently digested with an excess of BamHI and fractionated on agarose gels was purified and then selfligated in the presence of T4 DNA ligase. Following transformation of E. coli XL1-Blue MR (Stratagene), plasmid DNA was extracted and analyzed for the presence of HindIII, PacI, SwaI, DraI and BamHI restriction sites. The characteristics of the resulting SuperCos/HPS vector are illustrated in Figure $1 B$. The vector was subsequently used for the construction of two cosmid libraries with BamHI or Sau3A partially digested BHV-1 DNA. Because of the presence of $S w a \mathrm{I}$ and $P a c \mathrm{I}$ restriction sites closely flanking the BamHI cloning site, intact subgenomic inserts could be readily generated by a simple digestion of the cosmid recombinants (not shown). In addition, EcoRI and HindIII flanking sites could be used to identify the genomic regions that were included in the clones by comparing the restriction patterns obtained to the previously reported restriction maps (1). Because of its significantly improved MCS, the SuperCos/HPS vector will facilitate the characterization of large GC-rich DNA inserts. A similar strategy could be used to introduce GC-rich restriction sites for the analysis of ATrich DNA inserts.

\section{REFERENCES}

1.Mayfield, J.E., P.J. Good, V.H. VanOort, A.R. Campbell and D.E. Reed. 1983. Cloning and cleavage site mapping of DNA from bovine herpesvirus 1 (Cooper strain). J. Virol. 47:259-264.

2.Plummer, G., C.R. Goodheart, D. Henson and C.P. Bowling. 1969. A comparative study of the DNA density and behavior in tissue cultures of fourteen different herpesviruses. Virology 39:134-137.

3.Wahl, G.M., K.A. Lewis, J.C. Ruiz, B. Rothenberg, J. Zhao and G.A. Evans. 1987. Cosmid vectors for rapid genomic walking, restriction mapping, and gene transfer. Proc. Natl. Acad. Sci. USA 84:2160-2164.

We thank Johnny Basso for his kind assistance in the editing of this manuscript.
This work was funded by the Natural Sciences and Engineering Research Council of Canada (Grant No. STR0167587). Address correspondence to Claire Simard, Institut Armand-Frappier, Centre de recherche en virologie, 531 Boulevard des Prairies, Laval des Rapides, QC, Canada H7V $1 B 7$. Internet: claire_simard@iaf.uquebec.ca

Received 28 May 1997; accepted 1 July 1997.

\section{Sirinart Ananvoranich and Claire Simard \\ Institut Armand-Frappier \\ Laval des Rapides, QC, Canada}

\section{Duplication of a Region in the Multiple Cloning Site of a Plasmid Vector to En- hance Cloning-Mediated Addition of Restriction Sites to a DNA Fragment}

\author{
BioTechniques 23:814-816 (November 1997)
}

Cloning a DNA fragment requires that the ends of the insert be compatible with the ends of the vector, and maximum efficiency of the ligation is obtained when ends are cohesive. When adequate cohesive sites are not available, the vector and insert can be rendered blunt by using polymerases and/ or exonucleases, but blunt-end cloning is technically more difficult to carry out than cohesive-end cloning, especially when the insert is bigger than $1 \mathrm{~kb}$. Another approach is to add linkers to the blunt-ended insert (5). This involves synthesizing the two oligonucleotides in the linker, and the resulting DNA fragment will be delimited by identical restriction sites after digestion.

An alternative is to clone the fragment using polymerase chain reaction (PCR) while using primers that contain restriction sites (7). This method requires knowing the sequence at the ends of the fragment to be amplified and synthesizing the two primers; besides, unless special polymerases with proofreading activity are used in the PCR (Pfu, Vent ${ }^{\circledR}$, Deep Vent ${ }^{\mathrm{TM}}[\mathrm{New}$ England Biolabs, Beverly, MA, USA] etc.), this method is restricted to rather small DNA fragments (<2000 bp).

Another possibility is to clone the fragment in the multiple cloning site (MCS) of a vector and then release it with enzymes that cut at adjacent sites. A variant of this strategy is connected with the development of vectors having a special type of MCS that contains several couples of restriction sites generating compatible cohesive ends; the sites in every such couple are separated by other sites where the insert can be cloned. One vector of this type is pSP72 (Promega, Madison, WI, USA), and its MCS contains the sequence: XhoI-PvuII-HindIII-SphI-PstI-SalI-... As SalI and XhoI generate compatible 